\title{
High-scale SUSY breaking models in light of the BICEP2 result
}

\author{
Keisuke Harigaya, ${ }^{a}$ Masahiro Ibe, ${ }^{a, b}$ Koji Ichikawa, ${ }^{a}$ Kunio Kaneta ${ }^{a}$ \\ and Shigeki Matsumoto ${ }^{a}$ \\ ${ }^{a}$ Kavli IPMU (WPI), University of Tokyo, \\ Kashiwa, Chiba 277-8583, Japan \\ ${ }^{b}$ ICRR, University of Tokyo, \\ Kashiwa, Chiba 277-8582, Japan \\ E-mail: keisuke.harigaya@ipmu.jp, ibe@icrr.u-tokyo.ac.jp, \\ koji.ichikawa@ipmu.jp, kunio.kaneta@ipmu.jp, \\ shigeki.matsumoto@ipmu.jp
}

ABSTRACT: The large value of the tensor-to-scalar ratio in the cosmic microwave background radiation reported by the BICEP2 collaboration gives strong impact on models of supersymmetry (SUSY). The large ratio indicates inflation with a high-energy scale and thus a high reheating temperature in general, and various SUSY models suffer from the serious gravitino and Polonyi problems. In this article, we discuss a class of the high-scale SUSY breaking models which are completely free from those problems. With especially focusing on the dark matter relic abundance, we examine how the BICEP2 result narrows down the parameter space of the models, assuming the simplest chaotic inflation model. We find that the mass of the dark matter is predicted to be less than about $1 \mathrm{TeV}$ thanks to the non-thermal production in the early universe through the decay of abundant gravitinos produced after the reheating process. We also discuss implications in some details to dark matter searches at collider and indirect dark matter detection experiments.

KEYWORds: Supersymmetry Phenomenology

ARXIV EPRINT: 1403.5880 


\section{Contents}

1 Introduction 1

2 Chaotic inflation in supergravity 2

3 Dark matter candidates in gauginos 5

4 Conclusions $\quad 10$

\section{Introduction}

The Higgs boson mass of about $126 \mathrm{GeV}$ observed at the LHC experiment [1, 2] is rather heavier than the prediction of the conventionally studied minimal supersymmetric standard model (MSSM) with the superparticle masses of $\mathcal{O}(0.1-1) \mathrm{TeV}$. This rather large Higgs boson mass and the so far null-observation of the superparticles at the experiment seem to suggest models with rather heavy sparticles of $\mathcal{O}(10-100) \mathrm{TeV}$, i.e. the high-scale supersymmetry (SUSY) breaking models [3-7]. The models fitting the observed Higgs boson mass well are classified into the following two: (1) the models in which the masses of the scalars and the gauginos in the MSSM are close with each other, i.e. $m_{\text {gaugino }} \simeq m_{\text {scalar }}=\mathcal{O}(10$ 100) $\mathrm{TeV}$, and (2) those in which the gaugino masses are rather suppressed compared to the scalars, i.e. $m_{\text {gaugino }} \ll m_{\text {scalar }}=\mathcal{O}(10-100) \mathrm{TeV}$ [8]-[19]. ${ }^{1}$ These models are particularly favored combined with the cosmological gravitino problem [27]. That is, when the scalar masses are expected to be as large as the gravitino mass as in gravity mediation, the large scalar masses amount to a heavy gravitino, which decays well before the Big-Bang Nucleosynthesis starts [28-30].

Recently, the BICEP2 collaboration reported the first measurement of the tensor-toscalar ratio of $r=0.20_{-0.05}^{+0.07}$ in the cosmic microwave background (CMB) radiation [31]. Such a large ratio or a corresponding very large Hubble parameter during inflation, $H_{\mathrm{inf}} \sim$ $10^{14} \mathrm{GeV}$, gives strong impact on the SUSY models. In fact, as discussed in reference [32], such a high inflation scale gives an ordeal to models with $m_{\text {gaugino }} \simeq m_{\text {scalar }}$ like the conventional gravity mediation models. For models with $m_{\text {gaugino }} \simeq m_{\text {scalar }}$, the SUSY breaking field $Z$ is required to be neutral under any symmetry, and hence it is expected to have a large linear term in the Kähler potential, $K \simeq c Z+$ h.c., with $c$ being of order the Planck scale. In the presence of such a linear term, the scalar potential of the SUSY breaking field obtains a large linear term during inflation, with which the SUSY breaking field gets shifted to a large expectation value. As a result, the models with $m_{\text {gaugino }} \sim m_{\text {scalar }}$

\footnotetext{
${ }^{1}$ When $\tan \beta$, which is the ratio of the vacuum expectation values between up- and down-type Higgs doublets, is extremely close to 1, the observed Higgs boson mass allows us to have models with much higher SUSY breaking scales. See, for examples, references [20]-[26].
} 
suffer from a serious entropy problem, i.e. the Polonyi problem $[33],{ }^{2}$ without ingenious mechanisms [34-37]. For recent discussion on models with $m_{\text {gaugino }} \sim m_{\text {scalar }}$, see e.g. references [38-42].

On the other hand, the second class of the high-scale SUSY breaking models are free from the Polonyi problem, since it can be constructed without having a singlet SUSY breaking field [43]. The models are therefore consistent with the measured tensor-to-scalar ratio. In such models, the scalar bosons obtain SUSY breaking masses from the SUSY breaking sector via tree-level interactions of supergravity, and they are expected to be of the order of the gravitino mass. The gaugino masses are, on the other hand, generated at one-loop level mainly from so-called the anomaly mediated SUSY breaking (AMSB) contributions $[43,44]$. This class of models is also favored in view of the minimality since the models do not require additional mediation mechanisms.

In this article, we investigate the implications of the observed tensor-to-scalar ratio to the high-scale SUSY breaking models of the second class in more details. In our discussion, we take the simplest realization of the chaotic inflation model $[45,46]$ in supergravity $[47-$ 49]. We also focus on the models with the simplest origin of the $\mu$-term of the Higgs doublets [9-14], where it is generated by tree level interactions to the order parameter of the $R$-symmetry breaking [50-52], and hence it is of the order of the gravitino mass. ${ }^{3}$ As we will show, the reheating temperature in this class of models is rather high, which is favorable for successful leptogenesis scenario [53-55]. We will show that the neutralino dark matter density obtains sizable non-thermal contributions from the decay of the gravitino which is abundant for the high reheating temperature. As a result, we find that the mass of the neutralino dark matter is less than $1 \mathrm{TeV}$. We also discuss implications in some details to dark matter searches at collider and indirect dark matter detection experiments.

The article is organized as follows. In next section, we first briefly review the simplest realization of the chaotic inflation with a quadratic potential in supergravity, and discuss the most relevant operators for the decay of the inflaton in the high-scale SUSY breaking models (the pure gravity mediation (PGM) and the minimal Split SUSY models). We next discuss details of the neutralino dark matter paying particular attention to the nonthermal contributions to the dark matter density from the gravitino decay. The last section is devoted to conclusions.

\section{Chaotic inflation in supergravity}

Recently, the BICEP2 collaboration has reported an observation of the large scale B-mode in the $\mathrm{CMB}$, which is consistent with a large tensor-to-scalar ratio of $r=\mathcal{O}(0.1)$ in the primordial cosmic perturbation [31]. This large tensor-to-scalar ratio indicates a large inflation scale, which strongly suggests so-called the chaotic inflation model $[45,46]$. In

\footnotetext{
${ }^{2}$ It is worth notifying that this Polonyi problem cannot be solved by making the mass of the Polonyi field large by some interactions, because the linear term during inflation is very huge, and hence the SUSY breaking field $Z$ is shifted to the Planck scale during inflation at which the interactions responsible for the mass of the Polonyi field are ineffective [32].

${ }^{3}$ These models are now dubbed the pure gravity mediation (PGM) [9-14] or the minimal split SUSY models $[17,18]$. In what follows, the high-scale SUSY breaking models refer to these models.
} 
particular, the simplest chaotic inflation model with a quadratic potential of the inflaton $\phi$, $V=m^{2} \phi^{2} / 2$, where $m$ denotes the mass of the inflaton, well fits the BICEP2 data. In view of the minimality, we confine ourselves to the simplest chaotic inflation model and discuss how high the reheating temperature can be. It is worth emphasizing again that the second class of the high-scale SUSY breaking models we are discussing is one of the models which reflect the minimality. Here, we further assume that interactions between the inflaton sector and the MSSM fields are controlled by $\mathcal{O}(1)$ coefficients and the Planck scale if they have mass dimensions in the spirit of minimality. As we will see, these assumptions based on the minimality lead to a highly consistent scenario with very simple parametrization.

In supergravity, this form of the potential is realized by introducing two chiral multiplets $X$ and $\Phi$ that have the following Kähler potential and the superpotential [47],

$$
K=K\left(X X^{\dagger},\left(\Phi+\Phi^{\dagger}\right)\right)=X X^{\dagger}+\frac{1}{2}\left(\Phi+\Phi^{\dagger}\right)^{2}+\cdots, \quad W=m X \Phi
$$

where '.. ' denotes terms which are irrelevant for our discussion. Note here that the Kähler potential possesses a shift symmetry: $\Phi$ transforms as $\Phi \rightarrow \Phi+i \alpha$ under the symmetry with $\alpha$ being a real number. This symmetry plays a crucial role to realize the slow-roll inflation in the chaotic inflation scenario where the field value of the inflaton excesses the Planck scale. The inflaton filed $\phi$ is then identified with the imaginary part of the scalar component of $\Phi$. The shift symmetry is explicitly broken by the spurion field $m$, which transforms as $m \rightarrow m \Phi /(\Phi+i \alpha)$ under the symmetry. With the explicit breaking, the inflaton obtains the quadratic potential.

The power spectrum $\mathcal{P}_{\zeta}$ of the curvature perturbations $\zeta$ is given by [56], $\mathcal{P}_{\zeta}=$ $\left(m^{2} N_{e}\right) /\left(6 \pi^{2} M_{\mathrm{PL}}^{2}\right)$, where $N_{e}$ is the number of e-folding and $M_{\mathrm{PL}} \simeq 2.4 \times 10^{18} \mathrm{GeV}$ the Planck scale. From the observed power spectrum $\ln \left(10^{10} \mathcal{P}_{\zeta}\right) \simeq 3.1$ [57], we find that the mass of the inflaton is fixed to be $m \simeq 1.6 \times 10^{13} \mathrm{GeV} .{ }^{4}$ It should be noted that the tensorto-scalar ratio predicted in this model, $r=8 / N_{e}$, is consistent with the BICEP2 result for $N_{e} \simeq 50-60$.

Let us now discuss the decay of the inflaton. Here, we assume that interactions between the inflaton sector and the MSSM fields are controlled by $\mathcal{O}(1)$ coefficients and the Planck scale if they have mass dimensions. With these assumptions, we classify operators dictating the inflaton decay by their mass-dimensions. When the decay is induced by a dimension- $n$ Planck-suppressed operator $(n \geq 4)$, the reheating temperature $T_{R}$ is estimated to be

$$
T_{R} \simeq 0.26 \sqrt{\Gamma_{\phi} M_{\mathrm{PL}}} \simeq\left\{\begin{array}{ll}
3.2 c \times 10^{14}(\mathrm{GeV}) & (n=4) \\
2.1 c \times 10^{9}(\mathrm{GeV}) & (n=5) \\
1.4 c \times 10^{4}(\mathrm{GeV}) & (n=6)
\end{array} .\right.
$$

Here, the decay width of the inflaton is estimated as $\Gamma_{\phi} \simeq\left(c^{2} m / 8 \pi\right)\left(m / M_{\mathrm{PL}}\right)^{2 n-8}$ with $c$ being an $\mathcal{O}(1)$ coupling constant, assuming two-body decays. ${ }^{5}$ Let us note again that

\footnotetext{
${ }^{4}$ In the chaotic inflation model with a dynamically generated fractional power potential, the inflaton mass can be much higher [61,62], which leads to a higher reheating temperature.

${ }^{5}$ For the case of $n=4, T_{R}$ is estimated to be higher than the mass of the inflaton. In such a case, interactions between decay products and the inflaton affect the reheating process, and $T_{R}$ is modified from
} 


\begin{tabular}{|c|ccccccccccc|}
\hline & $\Phi$ & $X$ & $m$ & $H_{u}$ & $H_{d}$ & $Q$ & $\bar{u}$ & $\bar{d}$ & $L$ & $\bar{e}$ & $N$ \\
\hline$Z_{4}$ & 0 & 3 & 1 & 2 & 2 & 1 & 1 & 1 & 1 & 1 & 1 \\
$Z_{4 R}$ & 2 & 0 & 0 & 2 & 2 & 2 & 2 & 2 & 2 & 2 & 2 \\
\hline
\end{tabular}

Table 1. The charge assignments of the MSSM fields $\left(H_{u}, H_{d}, Q, \bar{u}, \bar{d}, L, \bar{e}\right)$, the right-handed neutrino fields $(N)$, and the fields in the inflaton sector $(\Phi, X)$.

the estimation depends on the choice of inflationary models and the assumption that the inflaton decay is controlled solely by the Planck scale.

For the case of $n=4$, the reheating temperature is so large that the universe is overclosed by the non-thermal dark matter production from the gravitino decay, as can be seen in equation (3.1). On the other hand, for the case of $n \geq 6$, the reheating temperature is so small that a scenario to generate the baryon asymmetry of the universe is limited. For $n=5$, on the other hand, the reheating temperature is consistent with the one required for successful thermal leptogenesis, namely $T_{R} \geq 2 \times 10^{9} \mathrm{GeV}[53-55,71]$. With this success, we focus on the case for $n=5$ in the following arguments. Furthermore, as we will see in next section, the dark matter mass is predicted to be $\mathcal{O}(1) \mathrm{TeV}$ to be consistent with the observed dark matter relic abundance thanks to the non-thermal production from the gravitino decay.

Here, it should be noted that the gravitinos are produced not only from scattering processes of the thermal bath but also from decay processes of the inflaton into the SUSY breaking sector. In particular, when the decay process is not controlled by symmetries, the branching ratios of the inflaton to the MSSM sector and the SUSY breaking sector are comparable, which eventually leads to overproduction of the gravitino and hence overproduction of dark matter [58-60]. To avoid such overproduction, we need to assume either some symmetries which suppress the branching ratio into the SUSY breaking sector or to assume fields in the SUSY breaking sector heavier than the inflaton.

For example, the first possibility can be realized by assuming a set of discrete symmetries given in table 1 which allows the inflaton decays into the MSSM fields via dimension 5 operators while the ones into the SUSY breaking sector are suppressed. ${ }^{6}$ In the model, the $Z_{4}$ symmetry is a discrete subgroup of the linear combination of $\mathrm{U}(1)_{\mathrm{Y}}$ and $\mathrm{U}(1)_{\mathrm{B}-\mathrm{L}}$ (so-called the fiveness), under which the MSSM fields have following charges;

$$
(Q, \bar{u}, \bar{e}): 1, \quad(L, \bar{d}):-3, \quad H_{u}:-2, \quad H_{d}: 2, \quad N: 5 .
$$

The $Z_{4}$ symmetry therefore guarantees the stability of the dark matter particle and the proton. ${ }^{7}$ Under this symmetry, the inflaton dominantly decays into the MSSM sector via

the above estimation [63]-[69, 70]. It turns out that $T_{R}$ is still at least as large as the mass of the inflaton, even if we take the effect of the interactions into account.

${ }^{6}$ The decay by the explicit breaking of the discrete symmetry is considered in reference [72], which leads to an additional small parameter to estimate the reheating temperature.

${ }^{7}$ The $Z_{4}$ symmetry is eventually broken into $Z_{2}$ so that the right-handed neutrinos obtain large Majorana masses. 

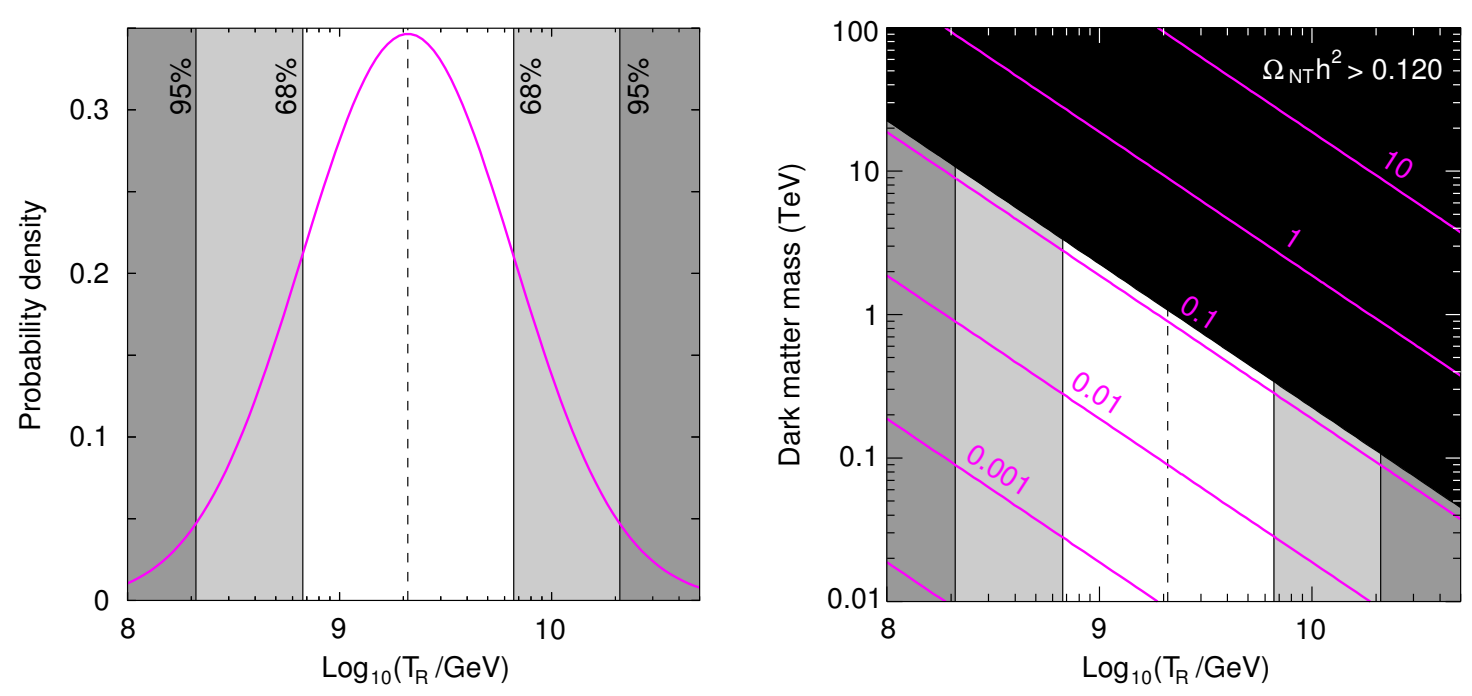

Figure 1. Left panel: the log-Gaussian probability density of $T_{R}$ deduced from the BICEP2 result. The light-gray and white regions are the ones favored at $95 \%$ and $68 \%$ confidence level, respectively. See text for more details. Right panel: contours of $\Omega_{\mathrm{NT}} h^{2}$ as a function of $T_{R}$ and $m_{\mathrm{DM}}$. The lightgray and white regions are the same as those in the left panel, while $\Omega_{\mathrm{NT}} h^{2}$ exceeds the observed abundance in the black region.

a dimension-5 operator ${ }^{8}$

$$
K \supset\left(c / M_{\mathrm{PL}}\right) X^{\dagger} L H_{u}+\text { h.c. },
$$

while the other modes are suppressed by more powers of the Planck scale.

In the latter possibility, that is, when the SUSY breaking sector is heavier than the inflaton, the inflaton decay into the SUSY breaking sector can be also suppressed [75]. In such a case, we do not have to impose additional discrete symmetries other than the $R$-symmetry which controls the $\mu$-term and the shift symmetry of the inflaton sector,and the inflaton decays through a dimension-5 operator,

$$
K \supset\left(c / M_{\mathrm{PL}}\right)\left(\Phi+\Phi^{\dagger}\right) H_{u} H_{d}+\text { h.c. }
$$

where the $R$ charge of the operator $H_{u} H_{d}$ vanishes. The consistency of the operator with the $R$ symmetry is characteristic of the special high-scale SUSY breaking models, namely the pure gravity mediation and the minimal split SUSY models.

\section{Dark matter candidates in gauginos}

In the high-scale SUSY breaking models (the PGM type), either the wino or the bino is predicted to be dark matter, and its relic abundance is determined by the sum of two different contributions. One is the contribution from the traditional thermal production

\footnotetext{
${ }^{8}$ Daughter particles of the inflaton are now charged under the $\mathrm{SU}(2)_{\mathrm{L}}$ symmetry. As is shown in references $[73,74]$, the daughter particles are then thermalized by the non-abelian gauge interaction instantaneously, and hence the estimation given in equation (2.2) is verified.
} 
$\left(\Omega_{\mathrm{TH}} h^{2}\right)[76,77]$ and the other is the non-thermal production from the gravitino decay $\left(\Omega_{\mathrm{NT}} h^{2}\right)$ [78, 79]. The latter contribution depends not only on the dark matter mass $\left(m_{\mathrm{DM}}\right)$ but also the reheating temperature $\left(T_{R}\right)$. When the temperature is higher, the more the gravitino is produced, and hence the contribution is larger. The non-thermal contribution is then estimated to be

$$
\Omega_{\mathrm{NT}} h^{2} \simeq 0.16\left(m_{\mathrm{DM}} / 300 \mathrm{GeV}\right)\left(T_{R} / 10^{10} \mathrm{GeV}\right) .
$$

In order to quantitatively discuss how the BICEP2 result affects the PGM type models, we assume that $T_{R}$ follows the log-Gaussian probability with its mean value and standard deviation being $\ln \left(2.1 \times 10^{9} \mathrm{GeV}\right)$ and $(\ln 10) / 2$, respectively. The use of such a probability is to take an $\mathcal{O}(1)$ ambiguity of $T_{R}$ into account, which is caused by e.g. an $\mathcal{O}(1)$ change of the coupling constant ' $\mathrm{c}$ ' in equation $(2.2)$. We show the probability density $P\left(\ln T_{R}\right)$ in the left panel of figure 1, where the probability itself is defined by $P\left(\ln T_{R}\right) d\left(\ln T_{R}\right)$. The light-gray and white regions are the ones favored by the BICEP2 result at $95 \%$ and $68 \%$ confidence level (C.L.), respectively, according to the log-Gaussian probability. The resultant non-thermal contribution $\Omega_{\mathrm{NT}} h^{2}$ is shown in the right panel as a function of $T_{R}$ and $m_{\mathrm{DM}}$. The light-gray and white regions are the same as those in the left panel. It can be seen that, when $T_{R} \simeq 2.1 \times 10^{9} \mathrm{GeV}$, the dark matter mass should be less than about $1 \mathrm{TeV}$ so that $\Omega_{\mathrm{NT}} h^{2}$ does not exceed the observed dark matter density, $\Omega^{(\mathrm{obs})} h^{2} \simeq 0.120$ [80].

The contribution to the dark matter relic abundance from the thermal production does not depend on $T_{R}$. Instead, it depends on the mass spectrum of the gauginos. In the PGM type models, the gauginos acquire their masses via anomaly mediated contributions and Higgsino threshold corrections [43, 44]. In addition, there are also other contributions when we consider well-motivated extension of the minimal SUSY standard model, i.e. extension with a vector-like matter field and/or a PQ sector [81]-[85]. We therefore treat the gaugino masses as free parameters. Then, remembering the fact that the mixing between the neutral wino and the bino after the electroweak symmetry breaking is much suppressed in the PGM type models, we have the following four possibilities for the spectrum: (I) the bino is the lightest SUSY particle (LSP) and the wino is the next-to-lightest SUSY particle (NLSP), (II) the bino is the LSP and the gluino is the NLSP, (III) the wino is the LSP and the bino is the NLSP, ${ }^{9}$ and (IV) the wino is the LSP and the gluino is the NLSP. In what follows, we discuss how the BICEP2 result gives impact on these possibilities by calculating the dark matter relic abundance, including the coannihilation between LSP and NLSP particles ${ }^{10}$ and the Sommerfeld effect [86, 87]-[90]. Based on obtained results, we also discuss some implications to gaugino searches at collider and indirect dark matter detection experiments.

\footnotetext{
${ }^{9}$ Honestly speaking, the charged wino should be called the NLSP, for the neutral wino is the LSP. In order to make our discussion simple, we have used the above designation.

${ }^{10}$ It is also possible to consider the coannihilation in which all the gauginos (the bino, the wino, and the gluino) participate. Since the effect of the BICEP2 result on this possibility can be easily read off from those on other possibilities, we omit to discuss it in this article. Here, it is also worth notifying that the chemical equilibrium between LSP and NLSP is always maintained thanks to decay, inverse-decay, and conversion processes mediated by the Higgsino, the squarks, and the sleptons, even if these particles are as heavy as $\mathcal{O}(100) \mathrm{TeV}$.
} 

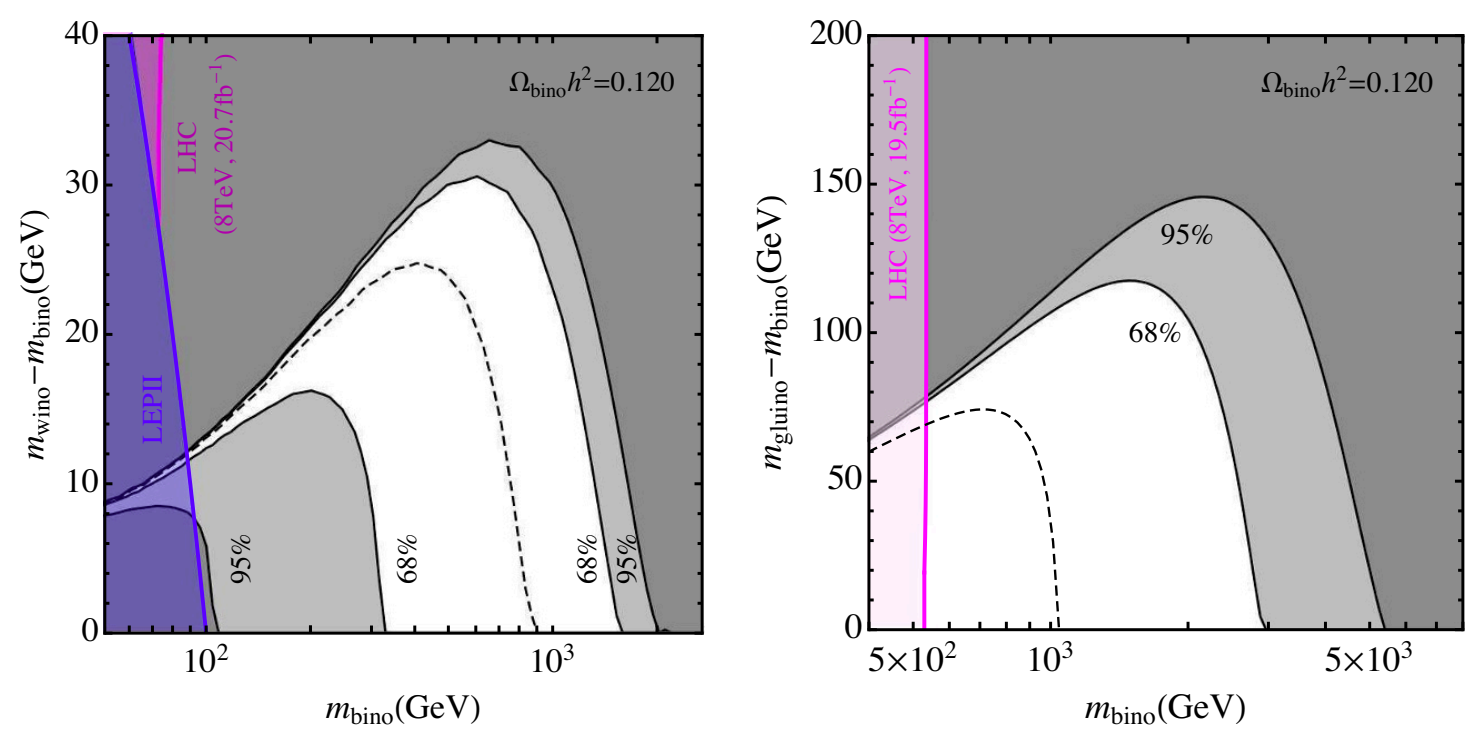

Figure 2. Left panel: the parameter region favored from the viewpoint of the dark matter relic abundance when the bino is the LSP and the wino is the NLSP. The light-gray and white regions are the same as those in figure 1. Limits from the LEP II and the LHC experiments at 95\% C.L. are also shown. Right panel: the parameter region favored from the viewpoint of the dark matter relic abundance when the bino is the LSP and the gluino is the NLSP. The light-gray and white regions are the same as those in the left panel. A limit from the LHC experiment at 95\% C.L. is also shown.

First, let us consider the case (I) where the bino is the LSP and the wino is the NLSP. The parameter region favored from the viewpoint of the dark matter relic abundance is shown in the left panel of figure 2 as a function of the bino mass $\left(m_{\text {bino }}\right)$ and the mass difference between the wino and the bino $\left(m_{\text {wino }}-m_{\text {bino }}\right)$. The light-gray and white regions are the same as those in figure 1, where the white (light-gray) region is favored by the BICEP2 result at $68 \%(95 \%)$ confidence level with assuming the simplest chaotic inflation model. It can be seen that the mass of the bino dark matter is at most around $1 \mathrm{TeV}$ with the $\mathcal{O}(10) \mathrm{GeV}$ mass difference thanks to the non-thermal contribution. Some limits obtained by collider experiments are also shown in the panel. The limit painted by blue comes from the LEP II experiment, which is obtained by the search for the wino pair production via the radiative return process [91]. The limit painted by purple is from the LHC experiment, which is obtained by the search for the production of neutral and charged winos [92]. Here we have assumed that the process provides three charged leptons with missing energy in its final state at $100 \%$ ratio. This assumption is verified in the most of the parameter region of the PGM type models, especially when the sleptons are somewhat lighter than the Higgsinos and/or the squarks. It is obvious that detection of $\mathcal{O}(10) \mathrm{GeV}$ soft leptons at $14 \mathrm{TeV}$ running of the LHC experiment will play an crucial role to detect the bino dark matter.

Next, we consider the case (II) where the bino is the LSP and the gluino is the NLSP. The parameter region favored by the dark matter relic abundance is shown in the right 

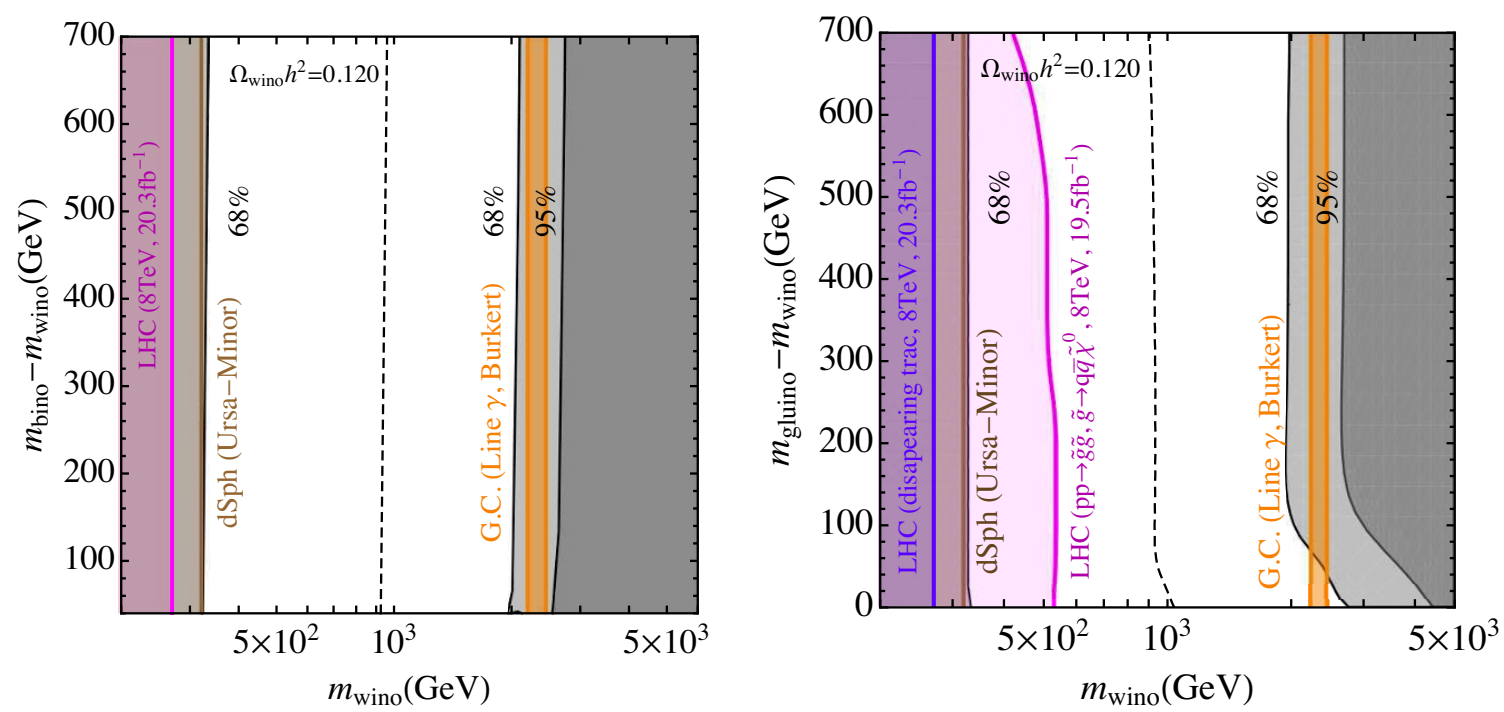

Figure 3. Left panel: the parameter region favored from the viewpoint of the dark matter relic abundance when the wino is the LSP and the bino is the NLSP. The light-gray and white regions are the same as previous figures. Limits from the LHC and the indirect dark matter detection (two kinds) experiments at $95 \%$ C.L. are also shown. Right panel: the parameter region favored from the viewpoint of the dark matter relic abundance when the bino is the LSP and the gluino is the NLSP. The light-gray and white regions are the same as those in the left panel. A limit from the LHC (two kinds) and the indirect dark matter detection (two kinds) experiments at 95\% C.L. are also shown.

panel of figure 2 as a function of the bino mass $\left(m_{\text {bino }}\right)$ and the mass difference between the gluino and the bino $\left(m_{\text {gluino }}-m_{\text {bino }}\right)$. The light-gray and white regions are the same as those in the left panel. Thanks to the non-thermal production again, the mass of the dark matter is predicted to be less than about $1 \mathrm{TeV}$ with the $\mathcal{O}(100) \mathrm{GeV}$ mass difference when $T_{R} \simeq 2.1 \times 10^{9} \mathrm{GeV}$. On the other hand, the dark matter mass is increased to a few $\mathrm{TeV}$ when the reheating temperature is somewhat suppressed, because the coannihilation process between gluinos is so efficient. Present limit from the LHC experiment is also shown in the plot as a pink region, which is obtained by the search for the gluino pair production associated with the initial state radiation(s) using $19.5 \mathrm{fb}^{-1}$ data at $8 \mathrm{TeV}$ running [93]. The limit will reach about $m_{\text {gluino }} \sim m_{\text {wino }} \sim 1 \mathrm{TeV}$ in near future using $100 \mathrm{fb}^{-1}$ data at $14 \mathrm{TeV}$ running [94], so that it can cover almost entire mass region of the bino dark matter when $T_{R} \simeq 2.1 \times 10^{9} \mathrm{GeV}$.

The wino is the LSP and the bino is the NLSP in the case (III). The parameter region favored by the dark matter relic abundance is shown in the left panel of figure 3 as a function of the wino mass $\left(m_{\text {wino }}\right)$ and the mass difference between the bino and the wino $\left(m_{\text {bino }}-m_{\text {wino }}\right)$. The light-gray and white regions are the same as those in previous figures. The dependence of the mass difference on the parameter region is very weak, because the dark matter relic abundance is almost governed by the wino self-annihilation and the non-thermal production. As in the case of the bino dark matter, the mass of the wino dark matter is predicted to be about $1 \mathrm{TeV}$ when $T_{R} \simeq 2.1 \times 10^{9} \mathrm{GeV}$. When the 
reheating temperature is somewhat suppressed, the predicted mass is shifted to a few $\mathrm{TeV}$, which is the value obtained when the abundance is determined only by the wino thermal production $[76,77]$.

Limits from the LHC and the indirect dark matter detection at the Fermi-LAT and the H.E.S.S. experiments are also shown as pink and orange regions, respectively. The LHC limit is from the search for the disappearing charged track caused by the charged wino decay inside inner detectors [95]. ${ }^{11}$ The wino mass up to $500 \mathrm{GeV}$ will be covered in near future by the search with $100 \mathrm{fb}^{-1}$ data at $14 \mathrm{TeV}$ running [97]. On the other hand, the limit from the indirect dark matter detection is obtained by observing gamma-rays from the galactic center (G.C.) [98] and dwarf spheroidal galaxies (dSphs) [99]. ${ }^{12}$ For the dSph observation, we use only a classical dSph (Ursa-Minor) to put a robust limit. The limit is not altered even if we include other classical dSphs. On the contrary, if we include ultra-faint dSphs in our analysis, the region $m_{\text {wino }}<0.4 \mathrm{TeV}$ and $2.13 \mathrm{TeV}<m_{\text {wino }}<2.53 \mathrm{TeV}$ is excluded. We omit to depict this limit in the plot, because the error of the dark matter profile inside each ultra-faint $\mathrm{dSph}$, namely the error of so called the $J$-factor, is still very large without assuming some relations between its profile and kinematic data $[101,102]$. In future, the parameter region $m_{\text {wino }}<0.77 \mathrm{TeV}$ and $1.91 \mathrm{TeV}<m<2.67 \mathrm{TeV}\left(m_{\text {wino }}<0.84 \mathrm{TeV}\right.$ and $1.85 \mathrm{TeV}<m_{\text {wino }}<2.7 \mathrm{TeV}$ ) will be covered by the Fermi-LAT experiment after 10 years (15 years) data taking. Here, we have assumed that the error of the $J$-factor is reduced to the level of the classical ones in future, namely $\Delta \log _{10}\left[J\left(0.5^{\circ}\right) /\left(\mathrm{GeV}^{2} \mathrm{~cm}^{-5} \mathrm{sr}\right)\right]=0.20$. In the G.C. observation, it is well known that the signal flux from the dark matter annihilation suffers from large systematic uncertainties due to our limited knowledge of the dark matter profile at the G.C. region [103]. We thus used the Burkert (cored) profile [104] instead of the NFW (cuspy) profile [105] in our analysis in order to put a robust limit.

Finally, we consider the case (IV) where the wino is the LSP and the gluino is the NLSP. The parameter region favored by the dark matter relic abundance is shown in the right panel of figure 3 as a function of the wino mass $\left(m_{\text {wino }}\right)$ and the mass difference between the gluino and the wino $\left(m_{\text {gluino }}-m_{\text {wino }}\right)$. The light-gray and white regions are the same as those in the left panel. The resultant region is almost the same as that for the case (III) except for the small one in which the wino is degenerate with the gluino and thus the gluino coannihilation process is efficient. Two limits from the LHC experiment are shown as blue and pink regions. The first one (which is the same as that in the left panel of figure 3) comes from the disappearing charged track search [95], while the other one (which is the same as that in the right panel of figure 2) is from the search for the gluino pair production [93]. These two searches will have complimentary roles at $14 \mathrm{TeV}$ running of the LHC experiment: the latter search will play an crucial role to explore the wino dark matter when the mass difference between the gluino and the wino is large enough, while

\footnotetext{
${ }^{11}$ The charged wino is highly degenerate with the neutral wino, so that it decays into a neutral wino by emitting a soft pion with its decay length of about $7 \mathrm{~cm}$. The mass difference between charged and neutral winos has already been calculated at full two-loop level [96].

${ }^{12}$ The indirect detection utilizing cosmic-ray anti-protons is potentially important, as clearly pointed out in reference [100], when systematic errors associated with the use of the diffusion equation are accurately evaluated. The limit on $m_{\tilde{w}}$ could be as strong as $m_{\tilde{w}}>500 \mathrm{GeV}$.
} 
the former one will be important when the mass difference is suppressed. The limit from the indirect dark matter detection at the Fermi-LAT and the H.E.S.S. experiments are shown as orange lines, which are the same as those in the left panel.

\section{Conclusions}

To conclude, the BICEP2 result has given strong impact on SUSY models, because the tensor-to-scalar ratio of $r \simeq 0.2$ means inflation occurred at some high scale. This fact indicates that the reheating temperature is also expected to be high in general, and we have to seriously consider the gravitino and the Polonyi problems in various SUSY models. The high-scale SUSY breaking models (the PGM type) are known to be completely free from the problems, so that the models become more and more attractive than before. In this article, we have therefore discussed the impact of the BICEP2 result on the models especially focusing on the dark matter relic abundance, adopting the simplest chaotic inflation model. Thanks to the non-thermal dark matter production in the early universe through the decay of the gravitinos produced just after the reheating process, the dark matter mass is predicted to be less than about $1 \mathrm{TeV}$ when $T_{R} \sim 2 \times 10^{9} \mathrm{GeV}$. This result encourages us very much, for the dark matter seems to be detected at collider or dark matter indirect detection experiments in the near future.

\section{Acknowledgments}

The authors thank T. T. Yanagida for useful discussion on the origin of the right-handed neutrino mass. This work is supported by Grant-in-Aid for Scientific Research from the Ministry of Education, Science, Sports and Culture (MEXT), Japan, No. 24740151 (M.I.), Nos. 23740169 \& 22244021 (S.M.), and also by World Premier International Research Center Initiative (WPI Initiative), MEXT, Japan. The work of K.H. and K.K. is supported by JSPS Research Fellowship for Young Scientists.

Open Access. This article is distributed under the terms of the Creative Commons Attribution License (CC-BY 4.0), which permits any use, distribution and reproduction in any medium, provided the original author(s) and source are credited.

\section{References}

[1] ATLAS collaboration, Observation of a new particle in the search for the Standard Model Higgs boson with the ATLAS detector at the LHC, Phys. Lett. B 716 (2012) 1 [arXiv: 1207.7214] [INSPIRE].

[2] CMS collaboration, Observation of a new boson at a mass of $125 \mathrm{GeV}$ with the CMS experiment at the LHC, Phys. Lett. B 716 (2012) 30 [arXiv:1207.7235] [INSPIRE].

[3] Y. Okada, M. Yamaguchi and T. Yanagida, Upper bound of the lightest Higgs boson mass in the minimal supersymmetric standard model, Prog. Theor. Phys. 85 (1991) 1 [INSPIRE].

[4] H.E. Haber and R. Hempfling, Can the mass of the lightest Higgs boson of the minimal supersymmetric model be larger than $m(Z)$ ?, Phys. Rev. Lett. 66 (1991) 1815 [INSPIRE]. 
[5] J.R. Ellis, G. Ridolfi and F. Zwirner, Radiative corrections to the masses of supersymmetric Higgs bosons, Phys. Lett. B 257 (1991) 83 [INSPIRE].

[6] G. Degrassi, S. Di Vita, J. Elias-Miro, J.R. Espinosa, G.F. Giudice et al., Higgs mass and vacuum stability in the Standard Model at NNLO, JHEP 08 (2012) 098 [arXiv:1205.6497] [INSPIRE].

[7] Y. Okada, M. Yamaguchi and T. Yanagida, Renormalization group analysis on the Higgs mass in the softly broken supersymmetric standard model, Phys. Lett. B 262 (1991) 54 [INSPIRE].

[8] J.D. Wells, PeV-scale supersymmetry, Phys. Rev. D 71 (2005) 015013 [hep-ph/0411041] [INSPIRE].

[9] M. Ibe, T. Moroi and T.T. Yanagida, Possible Signals of Wino LSP at the Large Hadron Collider, Phys. Lett. B 644 (2007) 355 [hep-ph/0610277] [INSPIRE].

[10] M. Ibe and T.T. Yanagida, The Lightest Higgs Boson Mass in Pure Gravity Mediation Model, Phys. Lett. B 709 (2012) 374 [arXiv:1112.2462] [INSPIRE].

[11] M. Ibe, S. Matsumoto and T.T. Yanagida, Pure Gravity Mediation with $m_{3 / 2}=10-100 \mathrm{TeV}$, Phys. Rev. D 85 (2012) 095011 [arXiv: 1202.2253] [InSPIRE].

[12] B. Bhattacherjee, B. Feldstein, M. Ibe, S. Matsumoto and T.T. Yanagida, Pure Gravity Mediation of Supersymmetry Breaking at the LHC, Phys. Rev. D 87 (2013) 015028 [arXiv: 1207.5453] [INSPIRE].

[13] J.L. Evans, M. Ibe, K.A. Olive and T.T. Yanagida, Universality in Pure Gravity Mediation, Eur. Phys. J. C 73 (2013) 2468 [arXiv:1302.5346] [InSPIRE].

[14] J.L. Evans, K.A. Olive, M. Ibe and T.T. Yanagida, Non-Universalities in Pure Gravity Mediation, Eur. Phys. J. C 73 (2013) 2611 [arXiv:1305.7461] [InSPIRE].

[15] B.S. Acharya, K. Bobkov, G.L. Kane, P. Kumar and J. Shao, Explaining the Electroweak Scale and Stabilizing Moduli in M-theory, Phys. Rev. D 76 (2007) 126010 [hep-th/0701034] [INSPIRE].

[16] L.J. Hall and Y. Nomura, Spread Supersymmetry, JHEP 01 (2012) 082 [arXiv:1111.4519] [INSPIRE].

[17] N. Arkani-Hamed, http://www.ift.uam.es/workshops/Xmas11/?q=node/2, in IFT Inaugural Conference, 2011.

[18] N. Arkani-Hamed, A. Gupta, D.E. Kaplan, N. Weiner and T. Zorawski, Simply Unnatural Supersymmetry, arXiv:1212.6971 [INSPIRE].

[19] A. Arvanitaki, N. Craig, S. Dimopoulos and G. Villadoro, Mini-Split, JHEP 02 (2013) 126 [arXiv: 1210.0555$]$ [INSPIRE].

[20] P.J. Fox, A.E. Nelson and N. Weiner, Dirac gaugino masses and supersoft supersymmetry breaking, JHEP 08 (2002) 035 [hep-ph/0206096] [INSPIRE].

[21] A. Hebecker, A.K. Knochel and T. Weigand, A Shift Symmetry in the Higgs Sector: Experimental Hints and Stringy Realizations, JHEP 06 (2012) 093 [arXiv:1204.2551] [INSPIRE].

[22] A. Hebecker, A.K. Knochel and T. Weigand, The Higgs mass from a String-Theoretic Perspective, Nucl. Phys. B 874 (2013) 1 [arXiv:1304.2767] [InSPIRE]. 
[23] J. Unwin, R-symmetric High Scale Supersymmetry, Phys. Rev. D 86 (2012) 095002 [arXiv: 1210.4936] [INSPIRE].

[24] L.E. Ibáñez, F. Marchesano, D. Regalado and I. Valenzuela, The Intermediate Scale MSSM, the Higgs Mass and F-theory Unification, JHEP 07 (2012) 195 [arXiv:1206.2655] [INSPIRE].

[25] L.E. Ibáñez and I. Valenzuela, The Higgs Mass as a Signature of Heavy SUSY, JHEP 05 (2013) 064 [arXiv:1301.5167] [INSPIRE].

[26] M. Ibe, S. Matsumoto and T.T. Yanagida, Flat Higgs Potential from Planck Scale Supersymmetry Breaking, Phys. Lett. B 732 (2014) 214 [arXiv:1312.7108] [INSPIRE].

[27] S. Weinberg, Cosmological Constraints on the Scale of Supersymmetry Breaking, Phys. Rev. Lett. 48 (1982) 1303 [INSPIRE].

[28] M. Kawasaki, K. Kohri and T. Moroi, Big-Bang nucleosynthesis and hadronic decay of long-lived massive particles, Phys. Rev. D 71 (2005) 083502 [astro-ph/0408426] [INSPIRE].

[29] M. Kawasaki, K. Kohri, T. Moroi and A. Yotsuyanagi, Big-Bang Nucleosynthesis and Gravitino, Phys. Rev. D 78 (2008) 065011 [arXiv:0804.3745] [INSPIRE].

[30] K. Jedamzik, Big bang nucleosynthesis constraints on hadronically and electromagnetically decaying relic neutral particles, Phys. Rev. D 74 (2006) 103509 [hep-ph/0604251] [INSPIRE].

[31] BICEP2 collaboration, P.A.R. Ade et al., Detection of B-Mode Polarization at Degree Angular Scales by BICEP2, Phys. Rev. Lett. 112 (2014) 241101 [arXiv:1403.3985] [INSPIRE].

[32] M. Ibe, Y. Shinbara and T.T. Yanagida, The Polonyi Problem and Upper bound on Inflation Scale in Supergravity, Phys. Lett. B 639 (2006) 534 [hep-ph/0605252] [INSPIRE].

[33] G.D. Coughlan, W. Fischler, E.W. Kolb, S. Raby and G.G. Ross, Cosmological Problems for the Polonyi Potential, Phys. Lett. B 131 (1983) 59 [InSPIRE].

[34] A.D. Linde, Relaxing the cosmological moduli problem, Phys. Rev. D 53 (1996) 4129 [hep-th/9601083] [INSPIRE].

[35] K. Nakayama, F. Takahashi and T.T. Yanagida, On the Adiabatic Solution to the Polonyi/Moduli Problem, Phys. Rev. D 84 (2011) 123523 [arXiv:1109.2073] [InSPIRE].

[36] K. Nakayama, F. Takahashi and T.T. Yanagida, Cosmological Moduli Problem in Low Cutoff Theory, Phys. Rev. D 86 (2012) 043507 [arXiv:1112.0418] [InSPIRE].

[37] K. Harigaya, M. Ibe, K. Schmitz and T.T. Yanagida, A Simple Solution to the Polonyi Problem in Gravity Mediation, Phys. Lett. B 721 (2013) 86 [arXiv:1301.3685] [INSPIRE].

[38] F. Brümmer and W. Buchmüller, A low Fermi scale from a simple gaugino-scalar mass relation, JHEP 03 (2014) 075 [arXiv:1311.1114] [INSPIRE].

[39] J.L. Feng, Z. Surujon and H.-B. Yu, Confluence of Constraints in Gauge Mediation: The 125 GeV Higgs Boson and Goldilocks Cosmology, Phys. Rev. D 86 (2012) 035003 [arXiv: 1205.6480] [INSPIRE].

[40] N. Okada, SuperWIMP dark matter and $125 \mathrm{GeV}$ Higgs boson in the minimal GMSB, arXiv: 1205.5826 [INSPIRE].

[41] O. Buchmueller, M.J. Dolan, J. Ellis, T. Hahn, S. Heinemeyer et al., Implications of Improved Higgs Mass Calculations for Supersymmetric Models, Eur. Phys. J. C 74 (2014) 2809 [arXiv: 1312.5233] [INSPIRE]. 
[42] N.E. Bomark and L. Roszkowski, The $3.5 \mathrm{keV} \mathrm{X-ray} \mathrm{line} \mathrm{from} \mathrm{decaying} \mathrm{gravitino} \mathrm{dark}$ matter, arXiv:1403.6503 [INSPIRE].

[43] G.F. Giudice, M.A. Luty, H. Murayama and R. Rattazzi, Gaugino mass without singlets, JHEP 12 (1998) 027 [hep-ph/9810442] [INSPIRE].

[44] L. Randall and R. Sundrum, Out of this world supersymmetry breaking, Nucl. Phys. B 557 (1999) 79 [hep-th/9810155] [INSPIRE].

[45] A.D. Linde, Chaotic Inflation, Phys. Lett. B 129 (1983) 177 [INSPIRE].

[46] A.D. Linde, Chaotic inflating universe, JETP Lett. 38 (1983) 176 [INSPIRE].

[47] M. Kawasaki, M. Yamaguchi and T. Yanagida, Natural chaotic inflation in supergravity, Phys. Rev. Lett. 85 (2000) 3572 [hep-ph/0004243] [INSPIRE].

[48] R. Kallosh, A. Linde, K.A. Olive and T. Rube, Chaotic inflation and supersymmetry breaking, Phys. Rev. D 84 (2011) 083519 [arXiv:1106.6025] [INSPIRE].

[49] A. Linde, Inflationary Cosmology after Planck 2013, arXiv:1402.0526 [INSPIRE].

[50] K. Inoue, M. Kawasaki, M. Yamaguchi and T. Yanagida, Vanishing squark and slepton masses in a class of supergravity models, Phys. Rev. D 45 (1992) 328 [INSPIRE].

[51] J.A. Casas and C. Muñoz, A Natural solution to the mu problem, Phys. Lett. B 306 (1993) 288 [hep-ph/9302227] [inSPIRE].

[52] G.F. Giudice and A. Masiero, A Natural Solution to the mu Problem in Supergravity Theories, Phys. Lett. B 206 (1988) 480 [InSPIRE].

[53] M. Fukugita and T. Yanagida, Baryogenesis Without Grand Unification, Phys. Lett. B 174 (1986) 45 [INSPIRE].

[54] W. Buchmüller, R.D. Peccei and T. Yanagida, Leptogenesis as the origin of matter, Ann. Rev. Nucl. Part. Sci. 55 (2005) 311 [hep-ph/0502169] [INSPIRE].

[55] S. Davidson, E. Nardi and Y. Nir, Leptogenesis, Phys. Rept. 466 (2008) 105 [arXiv:0802.2962] [INSPIRE].

[56] D.H. Lyth and A. Riotto, Particle physics models of inflation and the cosmological density perturbation, Phys. Rept. 314 (1999) 1 [hep-ph/9807278] [INSPIRE].

[57] Planck collaboration, P.A.R. Ade et al., Planck 2013 results. XXII. Constraints on inflation, arXiv:1303.5082 [INSPIRE].

[58] M. Kawasaki, F. Takahashi and T.T. Yanagida, Gravitino overproduction in inflaton decay, Phys. Lett. B 638 (2006) 8 [hep-ph/0603265] [InSPIRE].

[59] T. Asaka, S. Nakamura and M. Yamaguchi, Gravitinos from heavy scalar decay, Phys. Rev. D 74 (2006) 023520 [hep-ph/0604132] [INSPIRE].

[60] M. Endo, F. Takahashi and T.T. Yanagida, Inflaton Decay in Supergravity, Phys. Rev. D 76 (2007) 083509 [arXiv:0706.0986] [INSPIRE].

[61] K. Harigaya, M. Ibe, K. Schmitz and T.T. Yanagida, Chaotic Inflation with a Fractional Power-Law Potential in Strongly Coupled Gauge Theories, Phys. Lett. B 720 (2013) 125 [arXiv: 1211.6241] [INSPIRE].

[62] K. Harigaya, M. Ibe, K. Schmitz and T.T. Yanagida, Dynamical Chaotic Inflation in the Light of BICEPQ, Phys. Lett. B 733 (2014) 283 [arXiv:1403.4536] [INSPIRE]. 
[63] J. Yokoyama, Fate of oscillating scalar fields in the thermal bath and their cosmological implications, Phys. Rev. D 70 (2004) 103511 [hep-ph/0406072] [INSPIRE].

[64] J. Yokoyama, Can oscillating scalar fields decay into particles with a large thermal mass?, Phys. Lett. B 635 (2006) 66 [hep-ph/0510091] [INSPIRE].

[65] M. Bastero-Gil, A. Berera and R.O. Ramos, Dissipation coefficients from scalar and fermion quantum field interactions, JCAP 09 (2011) 033 [arXiv: 1008.1929] [INSPIRE].

[66] M. Bastero-Gil, A. Berera, R.O. Ramos and J.G. Rosa, General dissipation coefficient in low-temperature warm inflation, JCAP 01 (2013) 016 [arXiv:1207.0445] [INSPIRE].

[67] M. Drewes, On the Role of Quasiparticles and thermal Masses in Nonequilibrium Processes in a Plasma, arXiv:1012.5380 [INSPIRE].

[68] M. Drewes and J.U. Kang, The Kinematics of Cosmic Reheating, Nucl. Phys. B 875 (2013) 315 [arXiv: 1305.0267] [INSPIRE].

[69] K. Mukaida and K. Nakayama, Dynamics of oscillating scalar field in thermal environment, JCAP 01 (2013) 017 [arXiv:1208.3399] [INSPIRE].

[70] K. Mukaida and K. Nakayama, Dissipative Effects on Reheating after Inflation, JCAP 03 (2013) 002 [arXiv:1212.4985] [INSPIRE].

[71] W. Buchmüller, P. Di Bari and M. Plümacher, Leptogenesis for pedestrians, Annals Phys. 315 (2005) 305 [hep-ph/0401240] [INSPIRE].

[72] M. Kawasaki, M. Yamaguchi and T. Yanagida, Natural chaotic inflation in supergravity and leptogenesis, Phys. Rev. D 63 (2001) 103514 [hep-ph/0011104] [INSPIRE].

[73] S. Davidson and S. Sarkar, Thermalization after inflation, JHEP 11 (2000) 012 [hep-ph/0009078] [INSPIRE].

[74] K. Harigaya and K. Mukaida, Thermalization after/during Reheating, JHEP 05 (2014) 006 [arXiv: 1312.3097] [INSPIRE].

[75] K. Nakayama, F. Takahashi and T.T. Yanagida, Eluding the Gravitino Overproduction in Inflaton Decay, Phys. Lett. B 718 (2012) 526 [arXiv:1209.2583] [InSPIRE].

[76] J. Hisano, S. Matsumoto, M. Nagai, O. Saito and M. Senami, Non-perturbative effect on thermal relic abundance of dark matter, Phys. Lett. B 646 (2007) 34 [hep-ph/0610249] [INSPIRE].

[77] M. Cirelli, A. Strumia and M. Tamburini, Cosmology and Astrophysics of Minimal Dark Matter, Nucl. Phys. B 787 (2007) 152 [arXiv:0706.4071] [INSPIRE].

[78] T. Gherghetta, G.F. Giudice and J.D. Wells, Phenomenological consequences of supersymmetry with anomaly induced masses, Nucl. Phys. B 559 (1999) 27 [hep-ph/9904378] [INSPIRE].

[79] T. Moroi and L. Randall, Wino cold dark matter from anomaly mediated SUSY breaking, Nucl. Phys. B 570 (2000) 455 [hep-ph/9906527] [INSPIRE].

[80] Planck collaboration, P.A.R. Ade et al., Planck 2013 results. XVI. Cosmological parameters, arXiv:1303.5076 [INSPIRE].

[81] A.E. Nelson and N.J. Weiner, Extended anomaly mediation and new physics at 10-TeV, hep-ph/0210288 [INSPIRE]. 
[82] K. Hsieh and M.A. Luty, Mixed gauge and anomaly mediation from new physics at 10-TeV, JHEP 06 (2007) 062 [hep-ph/0604256] [INSPIRE].

[83] A. Gupta, D.E. Kaplan and T. Zorawski, Gaugomaly Mediation Revisited, JHEP 11 (2013) 149 [arXiv:1212.6969] [INSPIRE].

[84] K. Nakayama and T.T. Yanagida, Anomaly mediation deformed by axion, Phys. Lett. B 722 (2013) 107 [arXiv:1302.3332] [INSPIRE].

[85] K. Harigaya, M. Ibe and T.T. Yanagida, A Closer Look at Gaugino Masses in Pure Gravity Mediation Model/Minimal Split SUSY Model, JHEP 12 (2013) 016 [arXiv:1310.0643] [INSPIRE].

[86] J. Hisano, S. Matsumoto and M.M. Nojiri, Explosive dark matter annihilation, Phys. Rev. Lett. 92 (2004) 031303 [hep-ph/0307216] [INSPIRE].

[87] J. Hisano, S. Matsumoto, M.M. Nojiri and O. Saito, Non-perturbative effect on dark matter annihilation and gamma ray signature from galactic center, Phys. Rev. D 71 (2005) 063528 [hep-ph/0412403] [INSPIRE].

[88] S. Profumo and C.E. Yaguna, Gluino coannihilations and heavy bino dark matter, Phys. Rev. D 69 (2004) 115009 [hep-ph/0402208] [INSPIRE].

[89] A. De Simone, G.F. Giudice and A. Strumia, Benchmarks for Dark Matter Searches at the LHC, JHEP 06 (2014) 081 [arXiv:1402.6287] [INSPIRE].

[90] K. Harigaya, K. Kaneta and S. Matsumoto, Gaugino coannihilations, Phys. Rev. D 89 (2014) 115021 [arXiv:1403.0715] [INSPIRE].

[91] ALEPH collaboration, A. Heister et al., Search for charginos nearly mass degenerate with the lightest neutralino in $e^{+} e^{-}$collisions at center-of-mass energies up to 209-GeV, Phys. Lett. B 533 (2002) 223 [hep-ex/0203020] [INSPIRE].

[92] ATLAS collaboration, Search for direct production of charginos and neutralinos in events with three leptons and missing transverse momentum in $\sqrt{s}=8 \mathrm{TeV}$ pp collisions with the ATLAS detector, JHEP 04 (2014) 169 [arXiv: 1402.7029] [INSPIRE].

[93] CMS collaboration, Search for new physics in the multijet and missing transverse momentum final state in proton-proton collisions at $\sqrt{s}=8 \mathrm{TeV}$, JHEP 06 (2014) 055 [arXiv: 1402.4770] [INSPIRE].

[94] B. Bhattacherjee, A. Choudhury, K. Ghosh and S. Poddar, Compressed SUSY at $14 \mathrm{TeV}$ LHC, Phys. Rev. D 89 (2014) 037702 [arXiv:1308.1526] [INSPIRE].

[95] ATLAS collaboration, Search for charginos nearly mass degenerate with the lightest neutralino based on a disappearing-track signature in pp collisions at $\sqrt{s}=8 \mathrm{TeV}$ with the ATLAS detector, Phys. Rev. D 88 (2013) 112006 [arXiv:1310.3675] [INSPIRE].

[96] M. Ibe, S. Matsumoto and R. Sato, Mass Splitting between Charged and Neutral Winos at Two-Loop Level, Phys. Lett. B 721 (2013) 252 [arXiv:1212.5989] [INSPIRE].

[97] T. Yamanaka, http://www2.yukawa.kyoto-u.ac.jp/ ppp.ws/PPP2013/slides/YamanakaT.pdf, in Progress in Particle Physic, 2013.

[98] H.E.S.S. collaboration, A. Abramowski et al., Search for photon line-like signatures from Dark Matter annihilations with H.E.S.S, Phys. Rev. Lett. 110 (2013) 041301 [arXiv: 1301.1173] [INSPIRE]. 
[99] Fermi-LAT collaboration, M. Ackermann et al., Dark Matter Constraints from Observations of 25 Milky Way Satellite Galaxies with the Fermi Large Area Telescope, Phys. Rev. D 89 (2014) 042001 [arXiv: 1310.0828] [INSPIRE].

[100] A. Hryczuk, I. Cholis, R. Iengo, M. Tavakoli and P. Ullio, Indirect Detection Analysis: Wino Dark Matter Case Study, arXiv:1401.6212 [INSPIRE].

[101] G.D. Martinez, J.S. Bullock, M. Kaplinghat, L.E. Strigari and R. Trotta, Indirect Dark Matter Detection from Dwarf Satellites: Joint Expectations from Astrophysics and Supersymmetry, JCAP 06 (2009) 014 [arXiv:0902.4715] [INSPIRE].

[102] G.D. Martinez, A Robust Determination of Milky Way Satellite Properties using Hierarchical Mass Modeling, arXiv:1309.2641 [INSPIRE].

[103] F. Nesti and P. Salucci, The Dark Matter halo of the Milky Way, AD 2013, JCAP 07 (2013) 016 [arXiv: 1304.5127] [INSPIRE].

[104] A. Burkert, The Structure of dark matter halos in dwarf galaxies, IAU Symp. 171 (1996) 175 [astro-ph/9504041] [INSPIRE].

[105] J.F. Navarro, C.S. Frenk and S.D.M. White, The Structure of cold dark matter halos, Astrophys. J. 462 (1996) 563 [astro-ph/9508025] [INSPIRE]. 